\title{
Erratum to: Body Mass Index is Inversely Correlated with the Expanded CAG Repeat Length in SCA3/MJD Patients
}

Jonas Alex Morales Saute •

Andrew Chaves Feitosa da Silva •

Gabriele Nunes Souza • Aline Dutra Russo •

Karina Carvalho Donis • Leonardo Vedolin •

Maria Luiza Saraiva-Pereira •

Luis Valmor Cruz Portela • Laura Bannach Jardim

Published online: 3 January 2012

(C) Springer Science+Business Media, LLC 2011

\section{Erratum to: Cerebellum}

$$
\text { DOI 10.1007/s12311-011-0326-6 }
$$

"Unfortunately the wrong figure was published. Hereby the correct figure is published and we apologize for the inconvenience."
The online version of the original article can be found at http://dx.doi. org/10.1007/s12311-011-0326-6.

J. A. M. Saute $\cdot$ L. B. Jardim

Postgraduate Program in Medical Sciences,

Universidade Federal do Rio Grande do Sul,

Porto Alegre, Rio Grande do Sul, Brazil

\section{B. Jardim}

Department of Internal Medicine,

Universidade Federal do Rio Grande do Sul,

Porto Alegre, Rio Grande do Sul, Brazil

M. L. Saraiva-Pereira $\cdot$ L. V. C. Portela

Department of Biochemistry,

Universidade Federal do Rio Grande do Sul,

Porto Alegre, Rio Grande do Sul, Brazil

J. A. M. Saute

Neurology Service, Hospital de Clínicas de Porto Alegre (HCPA),

Porto Alegre, Rio Grande do Sul, Brazil
A. C. F. da Silva • G. N. Souza • A. D. Russo • K. C. Donis •

M. L. Saraiva-Pereira $\cdot$ L. B. Jardim

Medical Genetics Service,

Hospital de Clínicas de Porto Alegre (HCPA),

Porto Alegre, Rio Grande do Sul, Brazil

L. Vedolin

Neuroradiology Service, Hospital Moinhos de Vento,

Porto Alegre, Rio Grande do Sul, Brazil

L. B. Jardim $(\bowtie)$

Medical Genetics Service, Hospital de Clínicas de Porto Alegre (HCPA), Universidade Federal do Rio Grande do Sul,

Rua Ramiro Barcelos, 2.350,

90035-903 Porto Alegre, Rio Grande do Sul, Brazil

e-mail: ljardim@hcpa.ufrgs.br 
Fig. 1 BMI of SCA3/MJD

patients and its correlation

with (CAG)n. a BMI compari-

son of patients with SCA3/MJD

and controls corrected for ge-

values are given as means and

error bars represent SEM. b

CAG expanded repeat (CAG)n

simple correlation with BMI.

$* * p<0.01 ; * * * p<0.001$ a

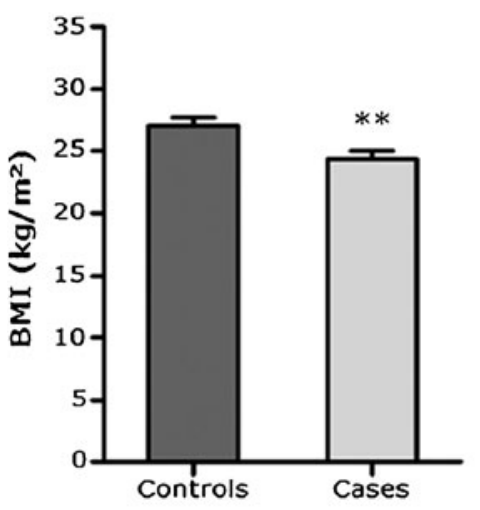

b

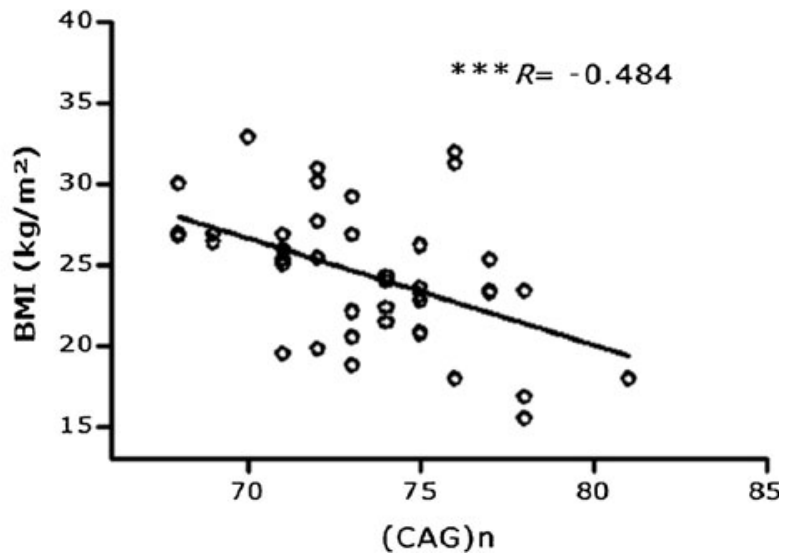

